\title{
Relationships between Fetal Alcohol Spectrum Disorder, Adverse Childhood Experiences, and Neurodevelopmental Diagnoses
}

\author{
Bradley J. Conant, Anne Sandstrom, Mariah Jorda, Marilyn G. Klug, Larry Burd* \\ Department of Pediatrics, University of North Dakota School of Medicine and Health Sciences, \\ Grand Forks, USA \\ Email: *larry.burd@und.edu
}

How to cite this paper: Conant, B.J., Sandstrom, A., Jorda, M., Klug, M.G. and Burd, L. (2021) Relationships between Fetal Alcohol Spectrum Disorder, Adverse Childhood Experiences, and Neurodevelopmental Diagnoses. Open Journal of Pediatrics, 11, 580-596.

https://doi.org/10.4236/ojped.2021.114054

Received: September 10, 2021

Accepted: October 26, 2021

Published: October 29, 2021

Copyright $\odot 2021$ by author(s) and Scientific Research Publishing Inc. This work is licensed under the Creative Commons Attribution International License (CC BY 4.0).

http://creativecommons.org/licenses/by/4.0/

\begin{abstract}
Objective: Children with fetal alcohol spectrum disorder (FASD) are overrepresented in early intervention programs, foster care, special education, juvenile corrections, and mental health services. In this study, we examine relationships between FASD and non-FASD controls for adverse childhood experiences (ACEs), and neurodevelopmental disorders. Methods: A chart review was conducted among patients seen at our clinic from 2010-2017 with data on FASD, ACEs, neurodevelopmental diagnoses, and foster or residential care placement available. Results: Relative risk for FASD was increased in patients with increased ACE scores $(\mathrm{RR}=5.08)$, increased numbers of neurodevelopmental diagnoses $(R R=2.36)$, and patients who have been in foster or residential care $(R R=9.53)$. FASD risk increased as ACE scores or the number of neurodevelopmental diagnoses increased. Patients with any ACEs were 3.96 times more likely to have FASD, and those with eight or more ACEs were 6.31 times more likely to have FASD than those with no ACEs. Patients with three or more neurodevelopmental diagnoses were 6.55 times more likely to have FASD than those with two or fewer diagnoses. Nine or more diagnoses increased the risk for FASD ten-fold $(R R=10.91)$. Conversely, patients diagnosed with FASD were more likely to have at least three ACEs (RR $=3.71)$, at least five neurodevelopmental diagnoses $(\mathrm{RR}=1.61)$, and high rates of previous foster or residential care placement $(R R=5.39)$. Conclusion: This study demonstrates that all children being considered for placement in foster care or residential should be screened for FASD.
\end{abstract}

\section{Keywords}

Fetal Alcohol Spectrum Disorder, Adverse Childhood Experiences, Foster Care, Residential Care, Developmental Diagnosis 


\section{Introduction}

Alcohol use among women of childbearing age in the United States continues to rise despite its well-established teratogenic effects and widespread recommendations against alcohol use during pregnancy [1]. More than half of all nonpregnant women of childbearing age report alcohol use during the previous month, and more than one in six report binge drinking in that time [2]. One in nine pregnant women also reports alcohol use during the previous month and one in twenty reports binge drinking [1] [2]. Over the course of a pregnancy, more than $30 \%$ of women report alcohol consumption, with greater than $8 \%$ reporting an episode of binge drinking [3]. The majority of pregnant women consuming alcohol did so during the first month $(22.5 \%)$, likely prior to pregnancy recognition, and only $2.7 \%$ of women report drinking throughout their pregnancy $(88 \%$ decrease) [3]. Another study reported that rates of prenatal alcohol exposure were $8.5 \%$ in the second month of pregnancy, decreased to $5.5 \%$ in the third month, and increased to $7.4 \%$ and $7.9 \%$ during the second and third trimesters respectively [3]. Considering these findings and the typical period of time during which a woman may be unaware she is pregnant, it is estimated that first-trimester exposure rates may be as high as $56 \%$ for all women and $78.9 \%$ for women with recent alcohol dependence [4].

Prenatal alcohol exposure poses a number of risks to the fetus, including the development of a fetal alcohol spectrum disorder (FASD) [5]. Four individual conditions with varying diagnostic criteria are classified under the term FASD: fetal alcohol syndrome (FAS), partial fetal alcohol syndrome, alcohol-related birth defects, and alcohol-related neurodevelopmental disorder (ARND) [6]. A diagnosis of FAS is made based on the presence of at least two characteristic facial anomalies, prenatal and/or postnatal growth deficiency, deficient brain growth, abnormal morphogenesis, or abnormal neurophysiology, and neurobehavioral impairment. Unfortunately, the physical features of FAS are often difficult to recognize, and consistency in diagnosis is generally dependent on the experience and expertise of the clinician. Partial fetal alcohol syndrome is diagnosed when there is documented prenatal alcohol exposure, at least two characteristic facial features, and neurobehavioral impairments. Partial fetal alcohol syndrome may be diagnosed in the absence of known prenatal alcohol exposure if growth deficiency, deficient brain growth, abnormal morphogenesis, or abnormal neurophysiology are present. Alcohol-related birth defects are diagnosed based on confirmed prenatal alcohol exposure and the presence of at least one major congenital malformation known to be the result of alcohol exposure [6]. ARND is the most commonly diagnosed FASD and requires confirmed prenatal alcohol exposure and neurobehavioral impairment with or without cognitive impairment [4] [6].

Multiple diagnostic criteria for FASD are currently utilized and comparisons between the criteria suggest that they have modest rates of agreement [7] [8]. Alternatively, these comparisons may demonstrate that the phenotype for FASD 
is broad and that the different diagnostic criteria each capture some unique portion of phenotypic variance.

The global prevalence of FASD has been estimated to be about $0.77 \%$ and approximately 630,000 children can be expected to be born with FASD each year [9]. Globally, this would suggest that an estimated 11 million children under age 18 , and an additional 14 million individuals between ages 19 and 40 have FASD [10]. Many regions have much higher rates of FASD, with estimates as high as $11.1 \%$ in South Africa including 13.5\% - 20.8\% in its Western Cape Province [6]. In the United States, the most conservative methods estimate the prevalence of FASD at $1 \%-5 \%$. Active case ascertainment methodologies result in prevalence estimates of $3.1 \%$ to $9.9 \%$ [11]. Notably, all of these prevalence estimates are still considered to be conservative due to challenges related to diagnosis.

Currently, diagnosis of fetal alcohol spectrum disorders is conducted by a multidisciplinary team and includes comprehensive neuropsychological assessment [6] [11] [12]. As a result of the inadequate availability of neuropsychological evaluation and dysmorphology services in community settings, the majority of diagnostic capacity falls to specialized clinics. These specialized clinics often rely on referrals from numerous sources, but limited diagnostic capacity and inadequate clinical recognition of individuals who warrant referral has resulted in high rates of undiagnosed or misdiagnosed patients [13] [14]. Many factors may be responsible for the lack of recognition, including lack of training in FASD, poor rates of screening, barriers to reporting of maternal alcohol use, legal concerns, lack of clear recommendations regarding screening, and confusion about the presence of comorbid health conditions [12] [13].

While the physical features associated with FAS and partial fetal alcohol syndrome are more overt signs of an FASD, the neurobehavioral and cognitive challenges faced by patients with ARND are more commonly the source of impairment across the lifespan [15] [16]. However, children with prenatal substance exposure often experience contact with the child welfare system due to child maltreatment [17]. Children diagnosed with FASD also have increased rates of placement in foster care due to neglect, abuse or family dysfunction [18]. This study suggests that the early post-natal environment of children with FASD likely influences their future developmental trajectory and both the severity of later impairments and the prevalence of neurodevelopmental disorders. This data suggests that both prenatal alcohol exposure and FASD may be associated with increased rates of exposure to adverse outcomes in childhood. Other research has demonstrated that childhood adversity is closely linked to ten adverse childhood experiences (ACEs). These were described in a landmark study by Felitti et al:: sexual abuse, emotional abuse, physical abuse, neglect, maternal abuse, and substance use in the household, mental illness in the household, divorced or separated parents, unloving family, and household member in prison [19]. Increasing numbers of ACEs have been shown to increase the likelihood of myriad health conditions, including severe obesity, lack of leisure-time physical activity, 
suicide attempts, alcoholism, illicit drug use, ischemic heart disease, cancer, diabetes, hepatitis, poor self-rated health [19].

While very useful, adult outcomes from exposure to ACEs could miss other manifestations of adversity earlier in a person's life when the identification of ACEs may also provide an opportunity to determine if early intervention could reduce the impact of ACEs across the lifespan. Recently, evidence of an association between ACEs and physical, mental, and developmental health in children was suggested [20]. Another study has demonstrated the presence of increased ACE scores in children with FASD [18]. Together these studies suggest that the interaction between pre and postnatal environments may have synergetic effects. Other studies have not identified this relationship [21]. Other research has suggested the exposure to some ACEs may result in differing outcomes compared to exposure to other ACEs. This study suggested that ACEs grouping deprivation may differ from ACEs more linked to threatening events [22]. FASD is a lifelong condition associated with a course of increasing clinical complexity often due to the extremely high rates of morbidity and mortality beginning in early childhood [10]. Thus increased risk of morbidity and mortality could be associated with complex interactions between prenatal exposures and the postnatal environment.

Numerous developmental disorders including attention-deficit/hyperactivity disorder (ADHD) and depression show marked increases in prevalence in FASD patients compared to the general population [16] [23] [24]. Almost all patients diagnosed with FASD will also have at least one additional diagnosis, with most patients having several comorbid conditions [16] [25]. Additionally, patients with an FASD are more likely than the general population to require educational support and encounter juvenile correctional systems [10] [26]. Mortality rates are also increased among people diagnosed with FASD. The increased mortality risk is not limited to the patient with FASD but also extends to mothers and siblings of those diagnosed with FASD [27] [28].

Increased rates of morbidity and mortality associated with FASD are the source of significant economic burden on society and the individual systems of care most likely to encounter individuals with FASD [29] [30]. The economic impact of FASD on families is huge. In North Dakota, the annual costs for parents with a child with FAS were estimated using a multiday cost diary at $\$ 17,400$ annually which was equal to $36.4 \%$ of the family gross annual income in 2009 [31]. Clinical experience and recent research demonstrated that FASD is associated with high rates of service usage and contact with professionals from numerous fields [24] [32]. Each encounter with a professional is an opportunity to identify a child who may have an undiagnosed FASD, ACEs or both and to implement a plan focused on diagnosis-informed care. Despite these individual and societal impacts of FASD, evidence suggests that the professionals most likely to encounter these patients are not adequately prepared to identify or treat them [33] [34]. The goal of this study was to examine the relationships between FASD, ACEs, and neurodevelopmental diagnoses. 


\section{Methods}

Chart reviews of patients seen at the North Dakota Fetal Alcohol Syndrome Center between 2010 and 2017 were used to identify the non-FASD cases and the cases with a diagnosis of FASD. The data used for this study was previously used to examine the relationship between FASD and ACEs [18]. In this study, we use this data to also examine the role of FASD and neurodevelopmental disorders. This center evaluates about 200 patients each year and provides follow-up for a large cohort of children and adults with FASD. A diagnosis of ARND was made based on the history of prenatal alcohol exposure, developmental assessment, and completion of the ARND Behavioral Checklist (ABC). Additional assessments were completed when necessary, including assessments of cognition, academic performance, attention, and mental health status. The $\mathrm{ABC}$ was developed in our clinic as part of an FASD surveillance and follow-up program as a tool to identify individuals with ARND. The reliability and validity of the $\mathrm{ABC}$ have been established against the proposed DSM-V criteria for ND-PAE [4]. Two recent studies have presented data on the sensitivity, specificity and accuracy of the $\mathrm{ABC}[35]$ [36].

The $\mathrm{ABC}$ is a 33 -item checklist completed by a caregiver who accompanies the patient to the visit. Each item is scored as either present or absent. After completion, the clinician reviews the checklist and scores any unreported items that have been observed during the interview. The number of behaviors that are scored as present are summed to calculate a total score for the checklist. The cut-off score for a positive $\mathrm{ABC}$ score differs by age group, with a score of 16 or greater considered diagnostic for individuals aged 3 - 6 years old and a score of 20 or greater considered diagnostic for individuals aged 7 years and older. ARND is not currently being diagnosed in individuals younger than three years old using this tool. In addition, each child also has a detailed diagnostic evaluation including an exposure assessment with dosimetry estimates, adaptive behavior testing, and $\mathrm{ADHD}$ testing. We also reviewed records for evidence of recent testing and physical examinations, past records documenting exposure, and results of the collection of any neurocognitive testing. Since nearly all patients have already been seen by multiple physicians, we do not routinely schedule another encounter. Where indicated by the referring physicians or parent or foster parent, we do schedule additional appointments as appropriate. These are for further assessment of vision, eating, toileting, sleep, growth or evaluation to see if medication is warranted. This data combined with the ABC score is used to diagnose FASD.

ACE scores were collected as part of a routine initial appointment at our clinic. The patient's caregiver is presented with a list of the ten ACEs and asked to identify which, if any, have been experienced by the patient. We also routinely collected information on two additional childhood experiences-placement in residential care or foster care-based on clinical experience and data suggesting that individuals with FASD are overrepresented in these populations. ACE 
scores collected during interviews were considered minimums because complete knowledge of our patient's postnatal environment is often unavailable.

Neurodevelopmental diagnoses were also collected as part of the typical initial appointment at our clinic. Information was collected through interviews with the patient and those attending the appointment with the patient. We routinely evaluated for 22 neurodevelopmental disorders: ADHD, learning disability, intellectual disability, cognitive impairment, verbal performance impairment, speech disorder, language disorder, tremors, tics, Tourette syndrome, obsessive-compulsive disorder, autism spectrum disorder, Rett's Syndrome, vision impairment, hearing impairment, oral comprehension deficits, memory impairment, anxiety, sleep disorder, enuresis, encopresis, and depression. When necessary, academic and medical records were referenced to evaluate neurodevelopmental diagnoses.

Patients were separated into two groups, those diagnosed with FASD and non-FASD controls. Inclusion criteria for the FASD group required the patient to be 21 years of age or under, diagnosed with FASD upon evaluation using the $\mathrm{ABC}$, and documentation of both ACEs and neurodevelopmental diagnoses. $\mathrm{Pa}$ tients were included in the control group if they were seen at the clinic in the stated time frame and had any neurodevelopmental disorder but did not meet the criteria for any FASD. Patients were excluded from the study if they were aged 22 years or older, did not have any neurodevelopmental diagnoses, or if prenatal alcohol exposure could not be confirmed.

\section{Statistical Analysis}

ACE scores and neurodevelopmental diagnoses were each divided into low, moderate, and high groups for the analysis. The relative risk of FASD for each group was estimated along with relative risk for FASD based on placement in foster or residential care. The reciprocal risk of moderate/high ACE score, moderate/high number of neurodevelopmental diagnoses, and foster or residential care placement was also calculated based on FASD diagnosis. Correlations between ACE scores, neurodevelopmental diagnoses, and foster or residential care placement were calculated. The relative risk for FASD based on ACE score or number of neurodevelopmental diagnoses was estimated for all values of these variables. All analyses were performed using SAS v 9.2.

\section{Results}

We identified 196 patients via chart review who were eligible for inclusion in the study based on the established criteria. There were 96 eligible patients $(49.0 \%)$ who received an FASD diagnosis and 100 eligible patients (51.0\%) who did not have FASD and were used as controls. The patients ranged in age from 3 to 20 years old (mean 8.85 years, $\mathrm{SD}=4.41$ ) and were predominantly male $(\mathrm{n}=127$, $64.8 \%)$. The most commonly reported races were American Indian/Alaskan Native $(\mathrm{n}=56,28.6 \%)$ and Caucasian $(\mathrm{n}=34,17.4 \%)$, though data on a single category of the race was unavailable for many of the participants (Table 1). The mean values for the ARND Behavioral Checklist, ACE score, and neurodeve- 
lopmental diagnoses for each group are also shown in Table 1. On average, patients with FASD scored 14.57 points higher on the ARND Behavioral Checklist, experienced 3.60 more ACEs, and had 2.17 more neurodevelopmental disorders than patients without FASD.

The relative risk for FASD based on ACE score grouping, number of neurodevelopmental diagnoses and previous placement in foster care or residential care, is displayed in Table 2. Patients with a moderate ACE score (3 - 6) were 4.78 times more likely to have FASD than those with fewer than 2 ACEs. Those with the highest ACE scores $(7-10)$ were 5.62 times more likely to have FASD. Overall, there is a five-fold increase in FASD risk for those who have experienced three or more ACEs $(R R=5.08)$. Patients with a moderate number of neurodevelopmental disorders $(5-8)$ were more than twice as likely to have FASD as those with fewer diagnoses $(\mathrm{RR}=2.12)$, and those with the most neurodevelopmental disorders $(9-13)$ were 3.53 times more likely to have FASD. Overall, children with five or more neurodevelopmental diagnoses were 2.36 times more likely to be diagnosed with FASD. Previous placement in residential care nearly doubled the likelihood of an FASD diagnosis $(\mathrm{RR}=1.95)$, and patients who have ever been in foster care were 8.73 times more likely to have FASD. A nearly ten-fold increase in FASD likelihood was found for patients who have been in residential care, foster care, or both $(\mathrm{RR}=9.53)$.

Figure 1 demonstrates the increasing risk for FASD as either the number of ACEs or the number of neurodevelopmental diagnoses increases. The RRs shown Table 1. Characteristics of FASD and non-FASD.

\begin{tabular}{|c|c|c|c|c|c|c|}
\hline & \multicolumn{3}{|c|}{ FASD $(\mathrm{N}=98)$} & \multicolumn{3}{|c|}{ Non-FASD $(\mathrm{N}=105)$} \\
\hline & $n$ & $\%$ & & $n$ & $\%$ & \\
\hline Male & 59 & 60.20 & & 72 & 68.57 & \\
\hline Female & 39 & 39.80 & & 33 & 31.43 & \\
\hline $\mathrm{AI} / \mathrm{AN}$ & 45 & 19.39 & & 13 & 14.29 & \\
\hline Caucasian & 19 & 45.92 & & 15 & 12.28 & \\
\hline Other & 34 & 34.69 & & 77 & 73.33 & \\
\hline Age $0-5$ & 26 & 26.53 & & 36 & 34.29 & \\
\hline $6-10$ & 36 & 36.73 & & 40 & 38.10 & \\
\hline \multirow[t]{2}{*}{$11-20$} & 36 & 36.73 & & 29 & 27.62 & \\
\hline & Mean & S.D. & Range & Mean & S.D. & Range \\
\hline Age & 9.306 & 4.680 & $2-20$ & 7.971 & 4.271 & $2-20$ \\
\hline ARND & 23.688 & 4.094 & $16-35$ & 9.120 & 4.441 & $1-19$ \\
\hline $\mathrm{ACE}$ & 5.323 & 2.573 & $0-10$ & 1.720 & 2.040 & $0-9$ \\
\hline Diagnoses & 6.667 & 2.347 & $1-13$ & 4.550 & 2.231 & $0-9$ \\
\hline
\end{tabular}


Table 2. Risk of FASD from ACE, diagnoses, and care.

\begin{tabular}{|c|c|c|c|c|c|c|c|}
\hline & \multicolumn{2}{|c|}{ FASD } & \multicolumn{2}{|c|}{ Non-FASD } & \multirow{2}{*}{$\mathrm{RR}$} & \multirow{2}{*}{$\begin{array}{c}95 \% \mathrm{CI} \\
\mathrm{LL}\end{array}$} & \multirow{2}{*}{$\begin{array}{c}95 \% \mathrm{CI} \\
\mathrm{UL}\end{array}$} \\
\hline & $n$ & $\%$ & $n$ & $\%$ & & & \\
\hline ACE $0-2$ & 14 & 15.38 & 77 & 84.62 & & & \\
\hline ACE $3-6$ & 50 & 73.53 & 18 & 26.47 & 4.8 & 2.9 & 7.9 \\
\hline ACE $7-10$ & 32 & 86.49 & 5 & 13.51 & 5.6 & 3.4 & 9.3 \\
\hline ACE 3+ & 82 & 78.10 & 23 & 21.90 & 5.1 & 3.1 & 8.3 \\
\hline Dx $0-4$ & 17 & 74.24 & 49 & 74.24 & & & \\
\hline Dx $5-8$ & 59 & 45.37 & 49 & 45.37 & 2.1 & 1.4 & 3.3 \\
\hline Dx $9-13$ & 20 & 90.91 & 2 & 9.09 & 3.5 & 2.3 & 5.4 \\
\hline Dx $5+$ & 79 & 60.77 & 51 & 39.23 & 2.4 & 1.5 & 3.6 \\
\hline No Care & 8 & 8.79 & 83 & 91.21 & & & \\
\hline Foster or Residential & 88 & 83.81 & 17 & 16.19 & 9.5 & 4.9 & 18.6 \\
\hline No Foster & 9 & 9.68 & 84 & 90.32 & & & \\
\hline Foster & 87 & 84.47 & 16 & 15.53 & 8.7 & 4.7 & 16.3 \\
\hline No Residential & 77 & 44.25 & 97 & 55.75 & & & \\
\hline Residential & 19 & 86.36 & 3 & 13.64 & 1.9 & 1.5 & 2.5 \\
\hline
\end{tabular}

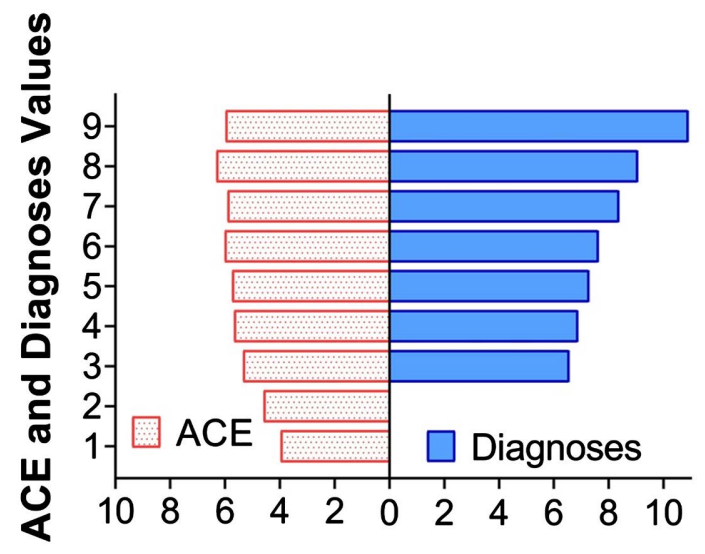

RR

Figure 1. The relative (RR) risk of FASD when the ACE score or number of diagnoses is greater or equal to a given value. All ACE RR shown are relative to an ACE score of zero.

for the number of diagnoses are all relative to having two or fewer diagnoses (zero or one diagnosis could not be used as a reference as there were no FASD children with zero diagnoses and only one with one diagnosis). Patients exposed to any ACEs were nearly four times more likely to have FASD compared to those who had an ACE score of zero ( $\mathrm{RR}=3.97)$. Those who had experienced eight or more ACEs were more than six times more likely to have FASD than those with no ACEs $(R R=6.31)$. Compared to patients with two or fewer diagnoses, individuals with three or more diagnoses were 6.5 times more likely to have FASD. 
Prenatal exposure resulting in FASD seems to account for most of the variance in total number of diagnoses. The likelihood of FASD increases as diagnoses increase, peaking at an eleven-fold increase in risk for patients with nine or more neurodevelopmental diagnoses $(\mathrm{RR}=10.91)$.

Patients with FASD were also found to be more likely to experience ACEs, receive a neurodevelopmental diagnosis, and be placed in foster care or residential care. Figure 2 demonstrates the bidirectional relationships between these interrelated factors and the correlations between them. Exposure to three or more ACEs increased the likelihood of a child having FASD more than five-fold compared to those experiencing fewer than three ACEs $(R R=5.08)$, and children with FASD were 3.71 times more likely to experience at least three ACEs than controls. Children with at least five neurodevelopmental disorders were more than twice as likely to have FASD as those with four or fewer diagnoses, and patients with FASD were $61 \%$ more likely to have at least five neurodevelopmental diagnoses. Placement in foster care or residential care increased the likelihood of a patient having FASD nearly ten-fold $(\mathrm{RR}=9.53)$, and children with FASD were more than five times more likely to be placed in foster or residential care than controls. ACE scores, neurodevelopmental diagnoses, and placement in foster care or residential care were all significantly correlated with one another. Correlations between the conditions were also significant (all $\mathrm{p}<0.001$ ) suggesting that if the risk is found in one area (e.g., many diagnoses), it is likely to be found in another area (e.g., foster or residential care).

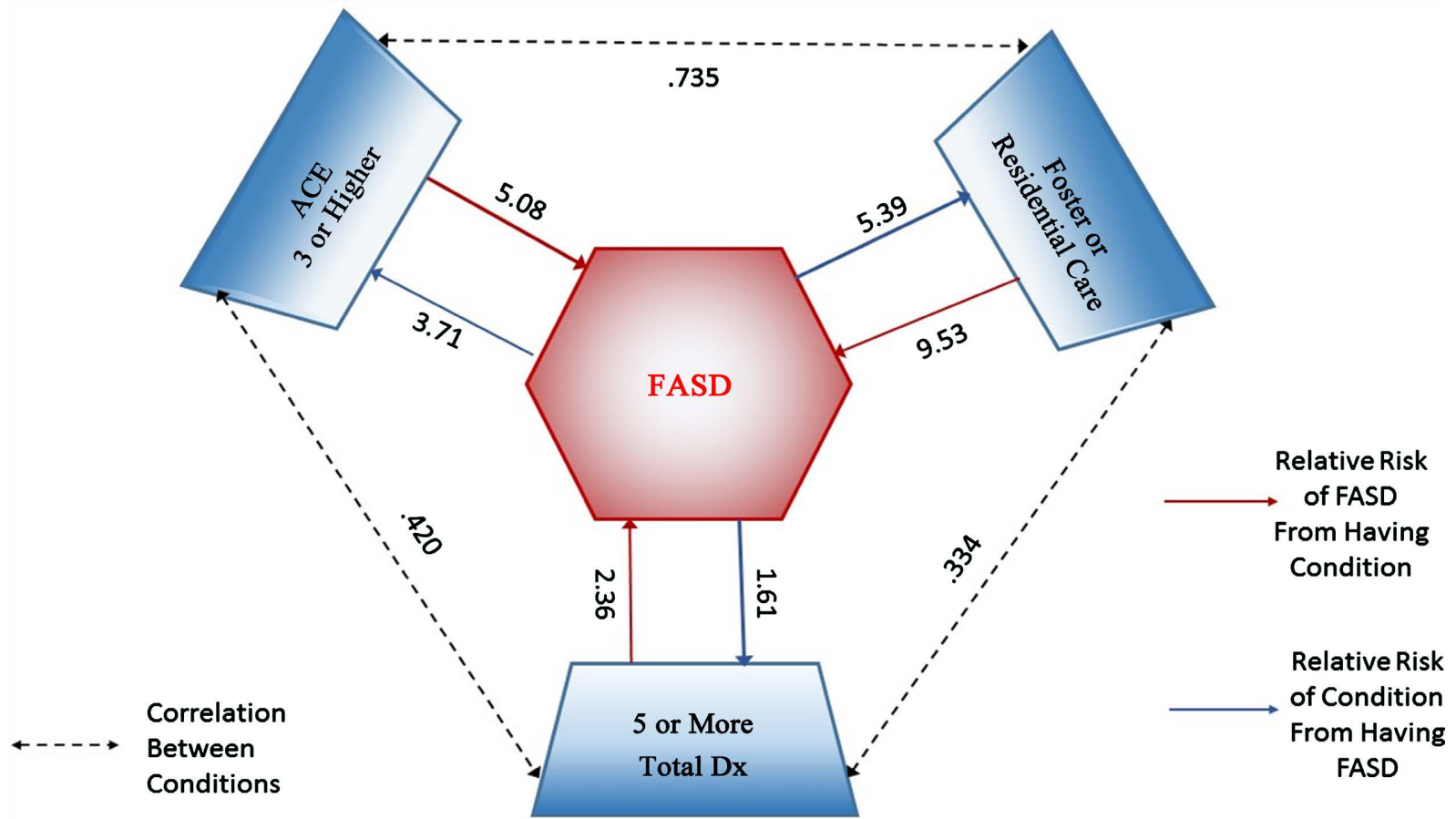

Figure 2. The diagram demonstrates the relationship between FASD and the ACE score, total number of neurodevelopmental diagnoses, and if the child was in foster or residential care (blue lines). The figure also shows the risk of FASD if the child has high ACE, total number of diagnoses, or lives in foster or residential care (red lines). 


\section{Discussion}

This study suggests that children with FASD experienced 3.6 more ACEs, and had 2.17 more neurodevelopmental disorders than patients without FASD. ACE scores, neurodevelopmental diagnoses, and placement in foster care or residential care were all significantly correlated (all $\mathrm{p}<0.001$ ). This demonstrates a close link between prenatal exposure, postnatal exposure to adversity, a diagnosis of FASD and placement in foster and/or residential care (primarily for children and adolescents with severe neurobehavioral disorders). This triple risk model is bidirectional. Children with high rates of ACEs are at increased risk for FASD and conversely, children with FASD are at high risk for exposure to ACEs. In our experience, this is especially likely for children with a history of multiple foster home placements. This suggests that early identification of ACEs could be used to identify children needing assessment for FASD. FASD intervention plans should emphasize strategies to decrease the risk for additional exposure to ACEs, and reduce additional placements in foster care and residential care. This should be a key role of social service agencies that very often have long-term custody of these infants and children. Thus, early diagnosis of FASD should encourage long-term planning by court teams to reduce the risk for additional exposure to these outcomes across childhood and adolescence.

FASD is considered the leading identifiable cause of intellectual disability in the United States with rates up to 23 times higher than the population at large [16] [23]. Rates of several other mental disorders are also significantly increased in the FASD population including psychotic disorder $(24.5 \times$ higher $)$, anxiety disorder $(11.2 \times)$, ADHD $(10 \times)$, reactive attachment disorder $(9.3 \times)$, oppositional defiant disorder $(4.9 \times)$, obsessive-compulsive disorder $(4.1 \times)$, depression $(4.0 \times)$, bipolar disorder $(3.2 \times)$, and post-traumatic stress disorder $(1.5 \times)$ [16]. The impacts of FASD also extend far beyond mental health concerns [24].

This makes FASD the most common identifiable cause of developmental disability, the leading known cause of neurobehavioral disorders in the United States, the most common diagnosis resulting in placement in foster care and residential care in the United States. FASD is also a leading cause of mortality for children and is very likely the leading cause of motility among young women of childbearing, age increasing mortality risk by 39-fold [28] [37].

FASD requires huge increases in cost of care for these children. These costs extend beyond the healthcare system. Individuals with FASD are hugely overrepresented in numerous special populations. Prevalence rates of FASD are 32 times greater among children in foster or residential care than the general population, and individuals with FASD are 9.22 times more likely to receive special education funding and 3.06 times more likely to repeat a grade [30] [38]. Overall, $61 \%$ of patients diagnosed with FASD have some form of disrupted school experience [39]. Life span prevalence rates for individuals with FASD are $60 \%$ for trouble with the law, 50\% for confinement, and $49 \%$ for repeated inappropriate sexual behaviors. Children with FASD are 19 times more likely to be in- 
carcerated in a given year and are 21 times more likely to develop a substance use disorder [24] [39].

These wide-ranging comorbidities culminate in extreme costs to society in terms of mortality, demands on service systems, and economics. Mortality rates are increased 4.9-fold for individuals with FASD and 2.6-fold for siblings of individuals with FASD [37]. Mothers of individuals with FASD face a 44.82-fold increase in mortality rate, with most deaths occurring in women younger than 50 years old [28]. The economic burden of FASD to society has recently been estimated to be $\$ 1.8$ billion annually in Canada and per person annual costs of $\$ 22,810$ - $\$ 24,308$ in the United States [29] [30]. The cost of care for a patient with FASD is $26 \%$ higher than autism, $87 \%$ higher than asthma in children, $13 \%$ higher than diabetes, and 56\% higher for epilepsy in adults [29].

Though the data are staggering, the morbidity and mortality associated with FASD need not be considered a foregone conclusion. Individuals diagnosed with FASD early and raised in a stable environment are 2- to 4 -fold more likely to avoid exposure to adverse childhood experiences later in life [37]. Additionally, several evidence-based methods have been established to benefit individuals with FASD and improve outcomes in various settings [40] [41]. Achieving the benefits of these interventions relies on early recognition and diagnosis, which remains a considerable weakness across many settings. Although pediatricians seem to be among the most likely professionals to recognize these patients, only $62 \%$ feel prepared to identify, $50 \%$ feel prepared to diagnose, and $34 \%$ feel prepared to manage treatment of children with FASD [33]. Among psychologists, $71 \%$ report lacking relevant training in FASD diagnosis, $42 \%$ underestimate the prevalence of FASD, and 65\% report being "very unprepared" or "somewhat unprepared" to identify a child with FASD compared to only $4 \%$ reporting feeling "very prepared" to identify these children [33]. In addition to pediatricians and psychologists, other professionals such as social workers, special education teachers, and neurologists who have the potential to recognize and aid in diagnosis of FASD are likely to encounter children with FASD due to the presence of comorbidities.

The results of the current study have the potential to substantially improve the ability of many of these professionals to identify individuals who may have FASD as well as to improve the holistic evaluation and management of the child's care if a diagnosis is made. The current study is the first the authors are aware of to demonstrate a bidirectional relationship between FASD and ACEs, neurodevelopmental diagnoses, and foster or residential care placement. Importantly, application of the results from the current study does not require knowledge of prenatal alcohol exposure. This helps to avoid a common barrier to FASD recognition, which involves asking the mother about alcohol use during pregnancy. Instead, a referral to a specialty center could be made, allowing a more experienced specialist to complete a formal evaluation. If a known history of prenatal alcohol exposure is present, a lower threshold for referral is likely 
warranted.

As shown in Figure 2, knowledge of any of the three factors can be used to predict risk for FASD, and an FASD diagnosis informs risk for ACEs, neurodevelopmental disorders, and foster or residential care placement. Professionals involved in the evaluation of any of these factors should therefore be aware of this relationship. For professionals involved in formal FASD evaluation, these results highlight the importance of holistic care and a proactive, diagnosis-informed treatment and management plan. Any time a patient is diagnosed with FASD, it is important to evaluate for ACEs and neurodevelopmental disorders. Evaluating these areas or referring to a specialist for evaluation helps to ensure that a more complete picture of the patient's impairment is captured. Evaluation for risk of foster or residential care placement is also crucial, especially considering the implications of the reauthorized Child Abuse Prevention and Treatment Act (CAPTA). This is potentially a source of services for families at risk of foster care placement. Thus, a wide range of services is available for families to prevent placement in foster care.

Information on ACEs, neurodevelopmental diagnoses, and foster or residential care placement will often be more readily available than formal FASD evaluations, especially for those who are most likely to encounter patients with undiagnosed FASD. For example, social workers are likely to have easily accessible knowledge of previous foster care or residential care placement and are well equipped to evaluate ACEs. Similarly, special education teachers and paraprofessionals are likely to have access to neurodevelopmental diagnoses through individualized education plans. Psychologists and psychiatrists are likely to have access to neurodevelopmental diagnoses through electronic medical records or their own evaluations and are highly trained to evaluate ACEs. Neurologists and pediatricians without training in FASD may also feel more comfortable evaluating any of these three interrelated factors than performing an FASD evaluation. When encountering a patient who has been in foster/residential care or a patient with many ACEs or neurodevelopmental diagnoses, all of these professionals should consider evaluating for FASD with a tool such as the ARND Behavioral Checklist or DSM-V proposed criteria for ND-PAE or referring to a specialty clinic for evaluation. Additionally, given one factor, professionals may consider assessing other dimensions to further evaluate their perception. For example, a pediatrician seeing a child who is in foster care could inquire about previous ACEs, or a neurologist seeing a patient with several neurodevelopmental diagnoses could ask if the child has previously been in foster or residential care.

The results of the current study and their relevance to numerous different professions represent a significant opportunity to increase diagnostic capability and access to treatment for FASD. The current difficulties in diagnosis and tendency toward conservative prevalence estimates make it very difficult to capture the full impact of FASD on individuals and society. Nevertheless, studies consistently demonstrate that FASD is a massive public health concern, with impacts 
that extend much further than several conditions that receive far more attention [29]. Establishing how different domains such as ACEs, neurodevelopmental disorders, and foster care placement impact FASD risk represents a significant step towards improving recognition of these patients by the many professionals they will encounter.

Several strategies are available to decrease the risk for FASD. The first is the adoption of routine screening for alcohol use among women of childbearing age. The second would be routine screening for alcohol use among pregnant women. The third is screening the children of women who have been in substance use treatment. Fourth would be to screen all children who enter neonatal intensive care units. All children should be screened as a part of well child care. Lastly, all siblings of a child diagnosed with FASD should be screened for FASD.

\section{Limitations}

There are several limitations associated with the current study. First, as a regional referral center, the patient population seen at our clinic is greatly influenced by geography and local populations. Although the participants in this study are representative of our overall patient population, a sample from other centers would likely differ significantly in terms of race and cultural norms and experiences. Second, ACEs and neurodevelopmental diagnoses were evaluated by recall during interviews. Therefore, both scores represent a summary of data that has, in many cases, been collected from several sources. No information on specific details or duration of adverse experiences was accounted for, which may influence outcomes. Additionally, ACE scores may be reported inaccurately by the individuals accompanying the patient. This may be due to incomplete knowledge of the postnatal environment by an adoptive or foster parent, or due to concerns of stigma or legal action by a biological parent. Third, although the reliability and validity of our diagnostic checklist have been established, we cannot be certain that all diagnoses are correct in the absence of a clinical biomarker. Finally, because all evaluations were performed at the same center and by the same clinician, any tendencies or biases in diagnosis would be expected to influence the data systemically.

Most of these limitations can and should be addressed through future research efforts. Larger, diverse multi-center replication studies should be developed to determine if these results are consistent across populations and clinicians. Additionally, these results should be investigated in the context of other commonly used diagnostic tools, including the DSM-V proposed criteria for ND-PAE. Most importantly, there should be efforts to incorporate these findings into clinical practice and evaluate their value in the context of various professions. Future research should also include identifying additional factors that increase the risk for FASD; identifying protective factors against FASD despite prenatal alcohol exposure; developing a potential method for identifying children under age three who show signs of FASD, increasing education about prenatal alcohol exposure 
for professionals in fields related to healthcare, education, corrections, and social work; promoting abstinence from substance use during pregnancy; and improving recognition and treatment for individuals with substance use disorders.

\section{Conclusion}

We conclude that ACEs, neurodevelopmental diagnoses, and foster or residential care placement are associated with increased risk for FASD, and conversely, individuals with FASD are at increased risk for exposure to ACEs, development of neurodevelopmental disorders, and placement in foster or residential care. A wide range of professionals is likely to encounter patients with FASD, including physicians, social workers, psychologists, special education teachers, and paraprofessionals. Increased rates of contact with judges, attorneys, social workers residential care staff and juvenile corrections systems demonstrate the need for further training and increased efforts to identify infants, children, and adolescents with FASD. Routine screening would be a useful strategy to identify these children.

\section{Funding}

The funding is provided by the REMS (Research Experience for Medical Students) Program at the University of North Dakota School of Medicine and Health Sciences.

\section{Conflicts of Interest}

The authors declare no conflicts of interest regarding the publication of this paper.

\section{References}

[1] Denny, C.H., Acero, C.S., Naimi, T.S., et al. (2019) Consumption of Alcohol Beverages and Binge Drinking among Pregnant Women Aged 18-44 Years-United States, 2015-2017. Morbidity and Mortality Weekly Report, 68, 365-368. https://doi.org/10.15585/mmwr.mm6816al

[2] Tan, C.H., Denny, C.H., Cheal, N.E., et al. (2015) Alcohol Use and Binge Drinking among Women of Childbearing Age-United States, 2011-2013. Morbidity and Mortality Weekly Report, 64, 1042-1046. https://doi.org/10.15585/mmwr.mm6437a3

[3] Ethen, M.K., Ramadhani, T.A., Scheuerle, A.E., et al. (2009) Alcohol Consumption by Women before and during Pregnancy. Maternal and Child Health Journal, 13, 274-285. https://doi.org/10.1007/s10995-008-0328-2

[4] Johnson, S., Moyer, C.L., Klug, M.G., et al. (2018) Comparison of Alcohol-Related Neurodevelopmental Disorder and Neurodevelopmental Disorder Associated with Prenatal Alcohol Exposure Diagnostic Criteria. Journal of Developmental and Behavioral Pediatrics, 39, 163-167. https://doi.org/10.1097/DBP.0000000000000523

[5] Stratton, K.R., Howe, C.J., et al. (1996) Fetal Alcohol Syndrome-Diagnosis, Epidemiology, Prevention, and Treatment. National Academy Press, Washington DC.

[6] Hoyme, H.E., Kalberg, W.O., Elliott, A.J., et al. (2016) Updated Clinical Guidelines 
for Diagnosing Fetal Alcohol Spectrum Disorders. Pediatrics, 138, 4256. https://doi.org/10.1542/peds.2015-4256

[7] Burd, L., Martsolf, J.T., Klug, M.G., et al. (2003) Diagnosis of Fas: A Comparison of the Fetal Alcohol Syndrome Diagnostic Checklist and the Institute of Medicine Criteria for Fetal Alcohol Syndrome. Neurotoxicology and Teratology, 25, 719-724. https://doi.org/10.1016/j.ntt.2003.07.015

[8] Coles, C.D., Gailey, A.R., Mulle, J.G., et al. (2016) A Comparison among 5 Methods for the Clinical Diagnosis of Fetal Alcohol Spectrum Disorders. Alcoholism: Clinical and Experimental Research, 40, 1000-1009. https://doi.org/10.1111/acer.13032

[9] Lange, S., Probst, C., Gmel, G., et al. (2017) Global Prevalence of Fetal Alcohol Spectrum Disorder among Children and Youth: A Systematic Review and Meta-Analysis. JAMA Pediatrics, 171, 948-956. https://doi.org/10.1001/jamapediatrics.2017.1919

[10] Popova, S., Lange, S., Shield, K., et al. (2019) Prevalence of Fetal Alcohol Spectrum Disorder among Special Subpopulations: A Systematic Review and Meta-Analysis. Addiction, 114, 1150-1172. https://doi.org/10.1111/add.14598

[11] May, P.A., Chambers, C.D., Kalberg, W.O., et al. (2018) Prevalence of Fetal Alcohol Spectrum Disorders in 4 Us Communities. JAMA, 319, 474-482.

[12] Montag, A.C. (2016) Fetal Alcohol-Spectrum Disorders: Identifying At-Risk Mothers. International Journal of Women's Health, 8, 311-323. https://doi.org/10.2147/IJWH.S85403

[13] Chasnoff, I.J., Wells, A.M. and King, L. (2015) Misdiagnosis and Missed Diagnoses in Foster and Adopted Children with Prenatal Alcohol Exposure. Pediatrics, 135, 264-270. https://doi.org/10.1542/peds.2014-2171

[14] Greenmyer, J.R., Popova, S., Klug, M.G., et al. (2020) Fetal Alcohol Spectrum Disorder: A Systematic Review of the Cost of and Savings from Prevention in the United States and Canada. Addiction, 115, 409-417. https://doi.org/10.1111/add.14841

[15] Burd, L., Klug, M.G., Martsolf, J., et al. (2003) Fetal Alcohol Syndrome: Neuropsychiatric Phenomics. Neurotoxicology and Teratology, 25, 697-705.

https://doi.org/10.1016/j.ntt.2003.07.014

[16] Weyrauch, D., Schwartz, M., Hart, B., et al. (2017) Comorbid Mental Disorders in Fetal Alcohol Spectrum Disorders: A Systematic Review. Journal of Developmental and Behavioral Pediatrics, 38, 283-291. https://doi.org/10.1097/DBP.0000000000000440

[17] Austin, A.E., Gest, C., Atkeson, A., et al. (2021) Prenatal Substance Exposure and Child Maltreatment: A Systematic Review. Child Maltreatment.

https://doi.org/10.1177/1077559521990116

[18] Kambeitz, C., Klug, M.G., Greenmyer, J., et al. (2019) Association of Adverse Childhood Experiences and Neurodevelopmental Disorders in People with Fetal Alcohol Spectrum Disorders (Fasd) and Non-Fasd Controls. BMC Pediatrics, 19, 498. https://doi.org/10.1186/s12887-019-1878-8

[19] Felitti, V.J., Anda, R.F., Nordenberg, D., et al. (1998) Relationship of Childhood Abuse and Household Dysfunction to Many of the Leading Causes of Death in Adults. The Adverse Childhood Experiences (Ace) Study. American Journal of Preventive Medicine, 14, 245-258. https://doi.org/10.1016/S0749-3797(98)00017-8

[20] Bright, M.A. and Thompson, L.A. (2018) Association of Adverse Childhood Experiences with Co-Occurring Health Conditions in Early Childhood. Journal of Developmental \& Behavioral Pediatrics, 39, 37-45. 
https://doi.org/10.1097/DBP.0000000000000514

[21] Flannigan, K., Kapasi, A., Pei, J., et al. (2021) Characterizing Adverse Childhood Experiences among Children and Adolescents with Prenatal Alcohol Exposure and Fetal Alcohol Spectrum Disorder. Child Abuse \& Neglect, 112, Article ID: 104888. https://doi.org/10.1016/j.chiabu.2020.104888

[22] Sheridan, M.A. and McLaughlin, K.A. (2014) Dimensions of Early Experience and Neural Development: Deprivation and Threat. Trends in Cognitive Sciences, 18, 580-585. https://doi.org/10.1016/j.tics.2014.09.001

[23] Burd, L. (2016) Fetal Alcohol Spectrum Disorder: Complexity from Comorbidity. The Lancet, 387, 926-927. https://doi.org/10.1016/S0140-6736(15)01346-X

[24] Popova, S., Lange, S., Shield, K., et al. (2016) Comorbidity of Fetal Alcohol Spectrum Disorder: A Systematic Review and Meta-Analysis. The Lancet, 387, 978-987. https://doi.org/10.1016/S0140-6736(15)01345-8

[25] Burd, L. and Popova, S. (2019) Fetal Alcohol Spectrum Disorders: Fixing Our Aim to Aim for the Fix. International Journal of Environmental Research and Public Health, 16, 3978. https://doi.org/10.3390/ijerph16203978

[26] Popova, S., Lange, S., Burd, L., et al. (2014) Canadian Children and Youth in Care: The Cost of Fetal Alcohol Spectrum Disorder. Child \& Youth Care Forum, 43, 83-96. https://doi.org/10.1007/s10566-013-9226-x

[27] Burd, L., Klug, M.G., Bueling, R., et al. (2008) Mortality Rates in Subjects with Fetal Alcohol Spectrum Disorders and Their Siblings. Birth Defects Research Part A: Clinical and Molecular Teratology, 82, 217-223. https://doi.org/10.1002/bdra.20445

[28] Li, Q., Fisher, W.W., Peng, C.Z., et al. (2012) Fetal Alcohol Spectrum Disorders: A Population Based Study of Premature Mortality Rates in the Mothers. Maternal and Child Health Journal, 16, 1332-1337. https://doi.org/10.1007/s10995-011-0844-3

[29] Greenmyer, J.R., Klug, M.G., Kambeitz, C., et al. (2018) A Multicountry Updated Assessment of the Economic Impact of Fetal Alcohol Spectrum Disorder: Costs for Children and Adults. Journal of Addiction Medicine, 12, 466-473.

https://doi.org/10.1097/ADM.0000000000000438

[30] Popova, S., Lange, S., Burd, L., et al. (2016) The Economic Burden of Fetal Alcohol Spectrum Disorder in Canada in 2013. Alcohol and Alcoholism (Oxford, Oxfordshire), 51, 367-375. https://doi.org/10.1093/alcalc/agv117

[31] Thanh, N.X. and Jonsson, E. (2009) Costs of Fetal Alcohol Spectrum Disorder in Alberta, Canada. The Canadian Journal of Clinical Pharmacology, 16, e90.

[32] Oh, S.S., Kim, Y.J., Jang, S.I., et al. (2020) Hospitalizations and Mortality among Patients with Fetal Alcohol Spectrum Disorders: A Prospective Study. Scientific Reports, 10, Article ID: 19512. https://doi.org/10.1038/s41598-020-76406-6

[33] Gahagan, S., Sharpe, T.T., Brimacombe, M., et al. (2006) Pediatricians' Knowledge, Training, and Experience in the Care of Children with Fetal Alcohol Syndrome. Pediatrics, 118, e657-e668. https://doi.org/10.1542/peds.2005-0516

[34] Wedding, D., Kohout, J., Mengel, M.B., et al. (2007) Psychologists' Knowledge and Attitudes about Fetal Alcohol Syndrome, Fetal Alcohol Spectrum Disorders, and Alcohol Use during Pregnancy. Professional Psychology: Research and Practice, 38, 208-213. https://doi.org/10.1037/0735-7028.38.2.208

[35] Burd, L., Klug, M.G. and Husark, K. (2021) Prevalence of Fetal Alcohol Spectrum Disorder and Screening in the Forensic Context: A Manual for Mental Health Practice. Springer International Publishing, Cham, 59-83.

https://doi.org/10.1007/978-3-030-73628-6_3 
[36] Klug, M.G., O'Connell, A.M., Palme, A., et al. (2021) A Validation Study of the Alcohol Related Neurodevelopmental Disorders Behavioral Checklist. Alcoholism: Clinical and Experimental Research, 45, 765-772.

https://doi.org/10.1111/acer.14570

[37] Schwartz, M., Hart, B., Weyrauch, D., et al. (2017) The Hidden Face of Fetal Alcohol Spectrum Disorder. Current Women's Health Reviews, 13, 96-102. https://doi.org/10.2174/1573404813666170418114243

[38] Brownell, M.D., de B Hanlon Dearman, A.C., Macwilliam, L.R., et al. (2013) Use of Health, Education, and Social Services by Individuals with Fetal Alcohol Spectrum Disorder. Journal of Population Therapeutics and Clinical Pharmacology, 20, e95-e106.

[39] Streissguth, A.P., Bookstein, F.L., Barr, H.M., et al. (2004) Risk Factors for Adverse Life Outcomes in Fetal Alcohol Syndrome and Fetal Alcohol Effects. Journal of Developmental \& Behavioral Pediatrics, 25, 228-238.

https://doi.org/10.1097/00004703-200408000-00002

[40] Bertrand, J. (2009) Interventions for Children with Fetal Alcohol Spectrum Disorders (Fasds): Overview of Findings for Five Innovative Research Projects. Research in Developmental Disabilities, 30, 986-1006. https://doi.org/10.1016/j.ridd.2009.02.003

[41] O’Connor, M.J., Laugeson, E.A., Mogil, C., et al. (2012) Translation of an Evidence-Based Social Skills Intervention for Children with Prenatal Alcohol Exposure in a Community Mental Health Setting. Alcoholism: Clinical and Experimental Research, 36, 141-152. https://doi.org/10.1111/j.1530-0277.2011.01591.x 